\title{
FITOPLANCTON DEL RÍO ALTO ANDINO "KAÑO" - TACNA (CORDILLERA SUROCCIDENTAL DEL PERÚ)
}

\author{
Juan Franco León ${ }^{1}$, Liduvina Sulca Quispe ${ }_{3}^{2}$ \\ César Cáceres Musaja ${ }^{3}$
}

\section{RESUMEN}

Se estudia la estructura fitoplanctónica del Río "Kaño" de la Cordillera Suroccidental del PerúTacna a 4,580 m.s.n.m. sobre la base de muestras colectadas mensualmente de enero a julio de 1999, siendo una zona colindante con los países Chile y Bolivia. El muestreo se realizó en la superficie, a media profundidad y del fondo.

Se registran cuatro divisiones que corresponde a CYANOPHYTA, EUGLENOPHYTA, BACILLARIOPHYTA Y CHLOROPHYTA, con una clara predominancia de las diatomeas sobre el resto de grupos.

Según las características observadas como tamaño pequeño de las células, niveles de organización, formación de cenobios, tipos de colonias y grupos taxonómicos predominantes, se puede deducir que este cuerpo de agua presenta condiciones tróficas para la crianza de peces introducidos, incrementando de esta manera la actividad diaria del poblador de la zona dedicado a la crianza de camélidos sudamericanos.

El presente trabajo tiene como objetivo principal aportar al conocimiento de cómo esta constituido la comunidad del fitoplancton y su influencia en la calidad del agua de este ecosistema acuático alto andino del departamento de Tacna.

\section{ABSTRACT}

The fitoplanctóniaca structure of Kaño river in the western south thighlands in Perú-Tacna 4580 m.snm) has been studied, based on samples collected monthly from january to july in 1999, bering an adjacent area to the chilean and Bolivian countries. the sampleing was done on the surface, to half depth and to the bostom of the river.

Four division corresponding to the CYANOPHYTA, EUGLENOPHYTA, BACILLARIOPHYTA AND CHLOROPHYTA have been registered, with a clear dominance of the diatomeas on the rest of groups.

According to the characteristics observed,such as: small size of the cells, organization levels, formation of monasteries, types of colories and groups predominant taxonómicos, one can deduce that this body - water presents trophical conditions grow introduced fish, increasing this way the daily activity of the resident inthe zone dedicated to the South American camelids groving.

This research work has as main objective to contribute to the knowledge of how the constituted community is cosntituted and its influence on the quality of the water from this aquatic ecosystem in the department of Tacna. 


\section{INTRODUCCIÓN}

En la Cordillera Suroccidental del Perú, se presentan entre 16 a 18 cuerpos de agua (lagunas, lagunillas, ríos y otros) muchos de los cuales se constituyen en importantes recursos hidrológicos para diferentes fines: producción de energía hidroeléctrica, consumo humano, riego agrícola, etc; pero muy pocos utilizados en acuicultura. No obstante, de este enorme potencial hidrológico no hay trabajos que caractericen el tipo de fitoplancton que presentan estos cuerpos de agua y que indiquen si pueden ser utilizados como un recurso piscicola en las actividades del poblador alto andino.

Químicamente, estos medios acuáticos de la zona se tipifican por presentar caracteristicas muy particulares, lo que condiciona de alguna manera también un tipo de fitoplancton caracteristico propio para cada medio.

El presente trabajo forma parte del estudio integrado de la diversidad biológica del fitoplancton de ecosistemas acuáticos alto andinos de Tacna, con el propósito de que estos puedan ser utilizados en forma racional por los pobladores de la zona y desarrollar más las fronteras vivas del sur.

En este sentido se plantea como objetivo principal: determinar la distribución taxonómica del fitoplancton y los principales parámetros limnológicos del medio acuático, con el fin de que sirvan como información básica en la utilidad pesquera.

\section{MATERIAL Y MÉTODOS}

El río Kaño se halla ubicado al Suroeste del lago Loriscota, en la pampa del mismo nombre, siendo un afluente de baja profundidad formado por filtraciones y acumulación de agua de bofedales. (Fig. 1)

Está entre las coordenadas geográficas de: LAT. SUR: $16^{\circ} 53^{\prime}$ y LONG. OESTE: $70^{\circ} 06^{\prime}$, a una altitud de 4,580 m.s.n.m.

La zona de muestreo se caracteriza por ser área de reposo y anidamiento de Fulica americana (choca - residente), Phoenicopterus chilensis (pariguana migratorio) y Oxyura ferruginea (zambullidor residente) en los bofedales que se disponen a los alrededores del río. La vegetación propia del lugar está representada por plantas acuáticas como: Potamogeton pectinatum, Elodea potamogeton y Ceratophyllum submerson, y entre las plantas terrestres tipicas se menciona a: Gnaphalium dombeyanum, Stipa ichu, Ephedra americana y Tagetes multiflora .

Se ejecutaron muestreos seriados, obteniéndose 25 muestras durante los meses de enero-julio 1999. Se siguió la metodología estandarizada de colecta con red de fitoplancton de $60-80 \mathrm{um}$, teniendo en cuenta la morfometría de los organismos, el que se analizó en el Laboratorio de Botánica de la U.N.J.B.G., empleándose un microscopio compuesto binocular con cámara fotográfica incorporada y cámara lúcida para esquemas. Para la identificación de las especies se utilizaron claves especializadas como la de Prescott (1978), Acleto (1998), Fernández (1999) y otros.

El análisis físico-químico del agua fue realizado en el Lab. de Química Analítica de la Universidad Nacional Jorge Basadre Grohmann Facultad de Ciencias. (Anexo I).

\section{RESULTADOS Y DISCUSIÓN}

\section{Reseña Taxonómica}

\section{CYANOPHYTA}

Clase Cyanophyceae

o. Chroococcales

Familia Chroococcaceae

Chroococcus limneticus Lemmermann (Fig. 1)

\section{O. HORMOGONALES}

Familia Oscillatoriaceae

Oscillatoriaceae

Oscillatoria proboscidea Gomont (Fig.2)

Oscillatoria formosa Bory. (Fig. 3)

Oscillatoria sancta Gomont (Fig. 4)

\section{O. NOSTOCALES}

Familia Nostocaceae

Microcystis aeruginosa Kutzing (Fig. 5)

Anabaena variabilis Kutzing (Fig. 6)

\section{BACILLARIOPHYTA}

Clase Bacillariophyceae

O. FRAGILARIALES

Familia Fragilariaceae

Fragilaria vaucheria Ehrenberg (Fig. 7) Synedra sp. Ehrenberg (Fig. 8) 


\section{O. NAVICULALES}

Familia Naviculaceae

Navicula cuspidata Kutzing (Fig. 9)

Pinnularia lata var. Rostata Breb. (Fig. 10)

Stauroneis anceps Ehr. (Fig. 11)

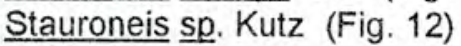

Caloneis sp. Smith. (Fig. 13)

Familia Gomphonemaceae

Gomphonema parvulum Ehrenberg. (Fig. 14)

Gomphonema sp. Ehrenberg (Fig. 15)

\section{O. EUNOTIALES}

Familia Achnathaceae

Cocconeis placentula Bory. (Fig. 16)

\section{O. EPITHEMIALES}

Familia Epithemiaceae

Rhopalodia gibberula Matter. (Fig. 17)

Ephitemia sp. Breb. (Fig. 18)

\section{O. NITZCHIALES}

Familia Nitzschiaceae

Nitzschia sp. Hasall (Fig. 19)

Nitzschia sp. Hasall (Fig. 20)

Hanzschia amphioxis Grunow. (Fig. 21)

\section{CHLOROPHYTA}

Clase Chlorophyceae

\section{O. ZIGNEMATALES}

Familia Desmidiaceae

Cosmarium decoratum. W. B \& G.S. West

(Fig. 22)

Cosmarium botrytis Bory. (Fig. 23)

Cosmarium sp. Ehr. (Fig. 24)

Cosmarium undulatum. Corda. (Fig. 25)

Closterium lebleinii. Kutz (Fig. 26)

Euastrum sp. Kirch (Fig. 27)

Familia Zygnemataceae

Spirogyra sp. Vauch. (Fig. 28)

\section{O. CHLOROCOCCALES}

Familia Oocystaceae

Ankistrodesmas falcatus Cord. (Fig. 29)

\section{EUGLENOPHYTA}

Clase Euglenophyceae

\section{O. EUGLENALES}

Familia Euglenaceae

Trachelomonas hispida Stein (Fig. 30)
Los grupos de organismos fotosintéticos son 4 que incluyen un total de 30 representantes. Las Bacillariophytas son las más abundantes, le siguen las Chorophytas, las Cyanophytas en último lugar. Al parecer esta distribución está en relación con los parámetros limnológicos del medio.

La División Cyanophyta está representada por 6 organismos (4 especies y 2 géneros), el género Oscillatoria es el que más frecuentemente se presenta manifestando formación de natas o capas sumergidas o flotantes, tomando un aspecto azul verdoso, al parecer esto no indicaría que se trata de un medio rico en nitritos y nitratos (Cole, 1988).

La División Bacillariophyta es la que mayor diversidad de especies presenta, entre las que predominan los géneros Synedra y Pinnularia. Varias de las especies descritas en el trabajo, son reportadas como indicadoras de agua con poca contaminación: como Cocconeis placentula, Navicula $\mathrm{sp}$, Pinnularia viridis, etc. lo que señalaria que las aguas del río Kaño permiten el desarrollo de otros organismos animales (Streble, 1990).

La División Chlorophyta representada con 8 especies y 2 géneros; en este grupo sobresalen las Desmidiaceas en forma muy abundante, sobre todo los géneros Cosmarium y Closterium, lo que difiere de alguna manera con lo reportado por Montoya (1993), que indica que especies de la Familia Desmidiaceae son indicadoras de ambientes ácidos del territorio alto andino central, puesto que en el cuerpo de agua de estudio, el Río Kaño, el pH es 7,79 siendo un medio alcalino.

En lo referente a los parámetros físico-químicos del río Kaño, estos se caracterizan por presentar valores típicos de aguas no salinas; así tenemos que la CE es $185.15 \mathrm{us} / \mathrm{cm}$, caracterizando esto a medios acuáticos cerrados, en los que su concentración de sales no aumenta con las precipitaciones fluviales (Moreno, 1996).

Las concentraciones de iones que presenta el medio en estudio, manifiesta una proporcionalidad tipo carbonato: $\mathrm{HCO}_{3}>\mathrm{SO}_{4}>\mathrm{Cl}$, en el que los carbonatos presentes como bicarbonatos exceden a los sulfatos y a los cloruros, caracterizan a un típico medio de aguas duras, donde deben estar presentes sedimentos calcárneos (Moreno, 1996).

En general se deduce que el agua es rica en oxígeno y cloro y presenta una diversidad de especies planctónicas y en las orillas presenta 
abundante vegetación herbácea. Estos medios también se caracterizan por presentar un mundo animal y vegetal muy variado que posibilita la existencia de organismos introducidos. Estas aguas son consideradas como beta mesosapróbicas, según los propios organismos indicadores de calidad del agua que presentan. (Streble \& Krauter, 1990).

\section{CONCLUSIONES}

1. De los resultados obtenidos en lo referente a la estructura del fitoplancton este se halla formado por cuatro divisiones: Cyanophyta, Euglenophyta, Bacillariaphyta y Chlorophyta. En el que las diatomeas tiene dominancia sobre el resto de organismos fotosintéticos.

2. Los Cyanophytas presentan una relativa diversidad de especies, las cuales se presentan como formas epífitas y planctónicas, siendo las especies

\section{REFERENCIAS BIBLIOGRÁFICAS}

COLE, G. 1988. Manual de Limnología. Ed. Hemisferio Sur. Buenos Aires - Argentina.

FRANCO, L. y COL. 1995. Fitoplancton de Aguas Contaminadas: Rio Torata-Moquegua. Ed. Rev. Ciencia y Desarrollo U.N.J.B.G., Nº 2, Tacna-Perú.

FERNÁNDEZ, A. 1999. Manual de las diatomeas Peruanas. Ed. Hortus Boanicus Truxillense. Trujillo-Perú.

GONZALES DE INFANTES.1988. El Plancton de las Aguas Continentales. Ed. Secretaria General de los Estados Americanos. Programa Regional de Desarrollo Cientifico y Tecnológico. Washington, USA. más comunes las que corresponde al género Oscillatoria.

3. Las Bacilloriophytas presentan relación entre su alta diversidad y el tipo de agua del río Kaño, puesto que este último es considerado como de aguas ligeramente duras. Así mismo, se presentan algunas especies indicadoras de aguas alcalinas como el caso de Cocconeis placentula.

4. Las Chorophytas se constituyen como el segundo grupo mejor representado en diversidad, sobre todo por la Desmidiaceas planctónicas, que predominan en el medio acuático.

5. Los parámetros físico-químicos del agua del río Kaño nos indican que se trata de aguas con pocas sales y que se pueden utilizar en la crianza de ciertos peces que se adapten con facilidad a estos medios acuáticos alto andinos.

MANGUIN, M. 1999. Contribución al conocimientos de las diatomeas de los Andes del Perú. Memorias del Museo Nacional de Historia Natural, Alemania.

MONTOYA, H. y BENAVENTE, M. 1993. Microalgas Altoandinas de la localidad de Raura, Lima. Ed. Rev. Arnaldoa. Vol 1 (4): 23-72. Trujillo-Perú

MORENO, T. 1996. Lagunas altoandinas del sur: características quimicas. Ed. Rev. Ciencia y Desarrollo, U.N.J.B.G. N ${ }^{0} 3$. Tacna-Perú.

STREBLE, H. y KRAUTER, D. 1990. Atlas de los microorganismos de agua Dulce. Ed. Omega S.A. Barcelona- España.

\section{ANEXO}

Parámetros quimicos del rio Kaño de la zona altiplänica occidental del Sur del Perú

\begin{tabular}{|l|c|}
\hline \multicolumn{1}{|c|}{ Parámetro } & Zona de muestreo \\
\hline $\mathrm{T}^{\circ}\left({ }^{\circ} \mathrm{C}\right)$ & 9 \\
\hline $\mathrm{OD}(\mathrm{mg} / \mathrm{L})$ & 11.3 \\
\hline $\mathrm{pH}$ & 8 \\
\hline $\mathrm{uS} \mathrm{cm}{ }^{-1}$ & 185.16 \\
\hline $\mathrm{CO}_{3} \mathrm{meg} / \mathrm{L}$ & 0.57 \\
\hline $\mathrm{Cl} \mathrm{meg/L}$ & 0.37 \\
\hline $\mathrm{SO}_{4} \mathrm{meg} / \mathrm{L}$ & 0.40 \\
\hline $\mathrm{Suma}$ de Aniones & \\
\hline $\mathrm{Ca}{ }^{+} \mathrm{meg} / \mathrm{L}$ & 0.49 \\
\hline $\mathrm{Mg}{ }^{+} \mathrm{meg} / \mathrm{L}$ & 0.35 \\
\hline $\mathrm{Na}+\mathrm{meg} / \mathrm{L}$ & 1.14 \\
\hline
\end{tabular}




\section{LÁMINAS}

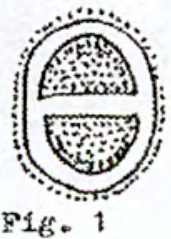

Pis. 2
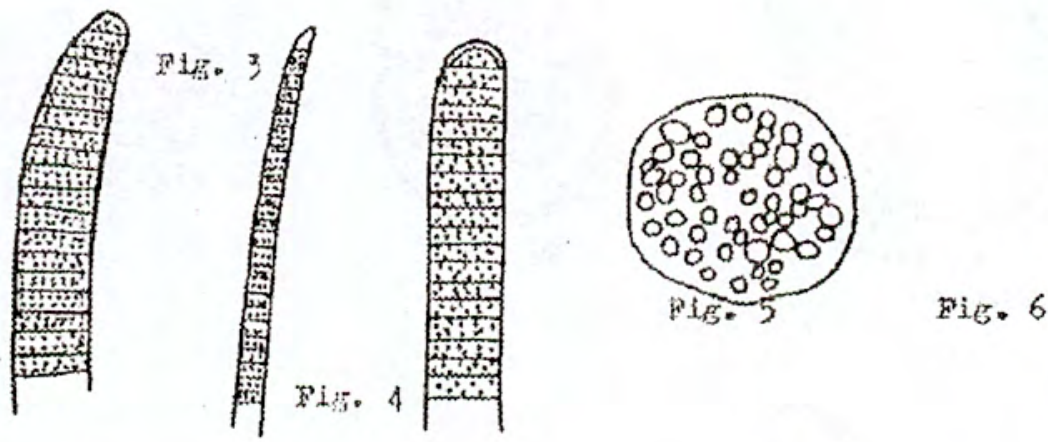

星
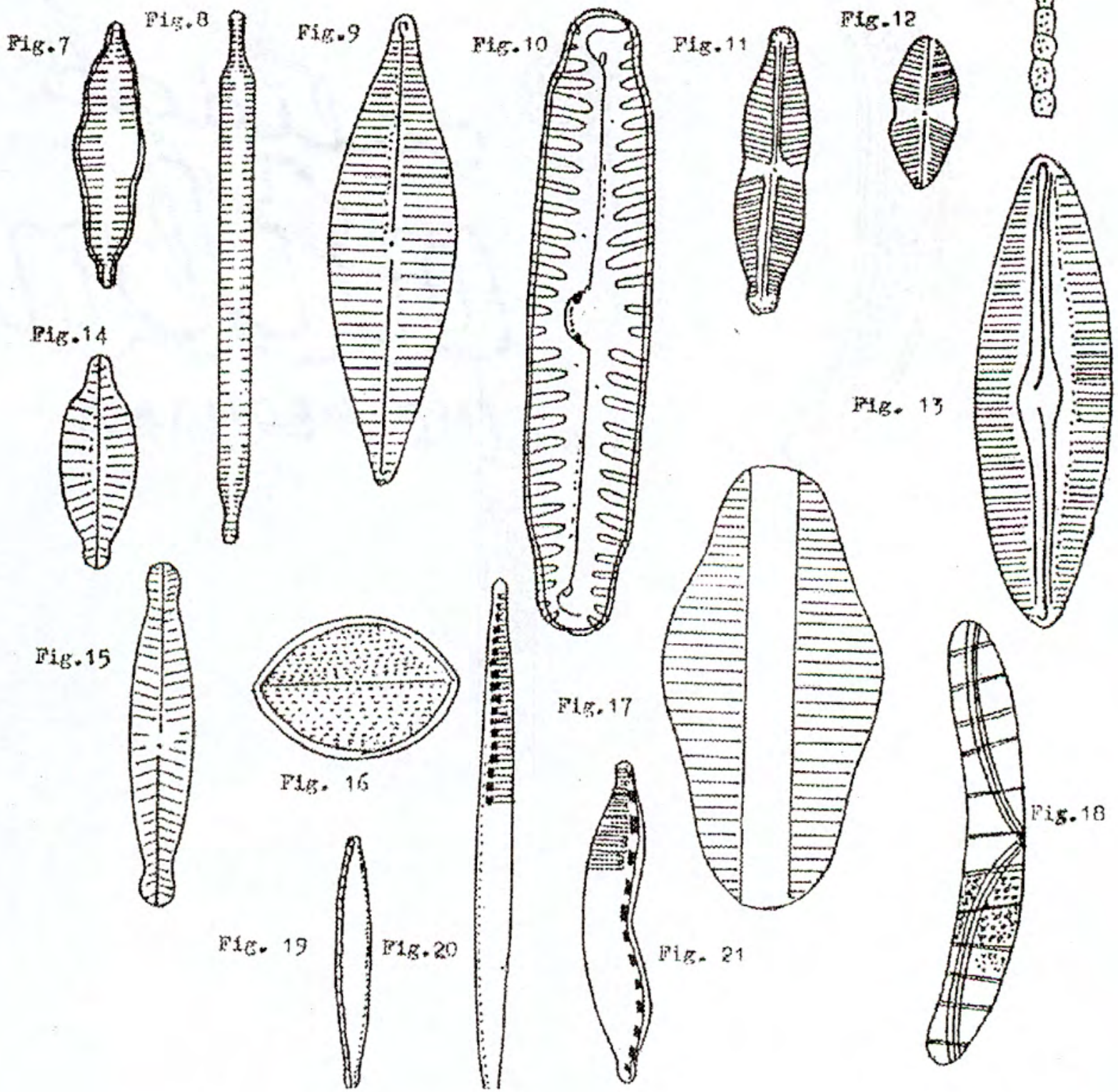

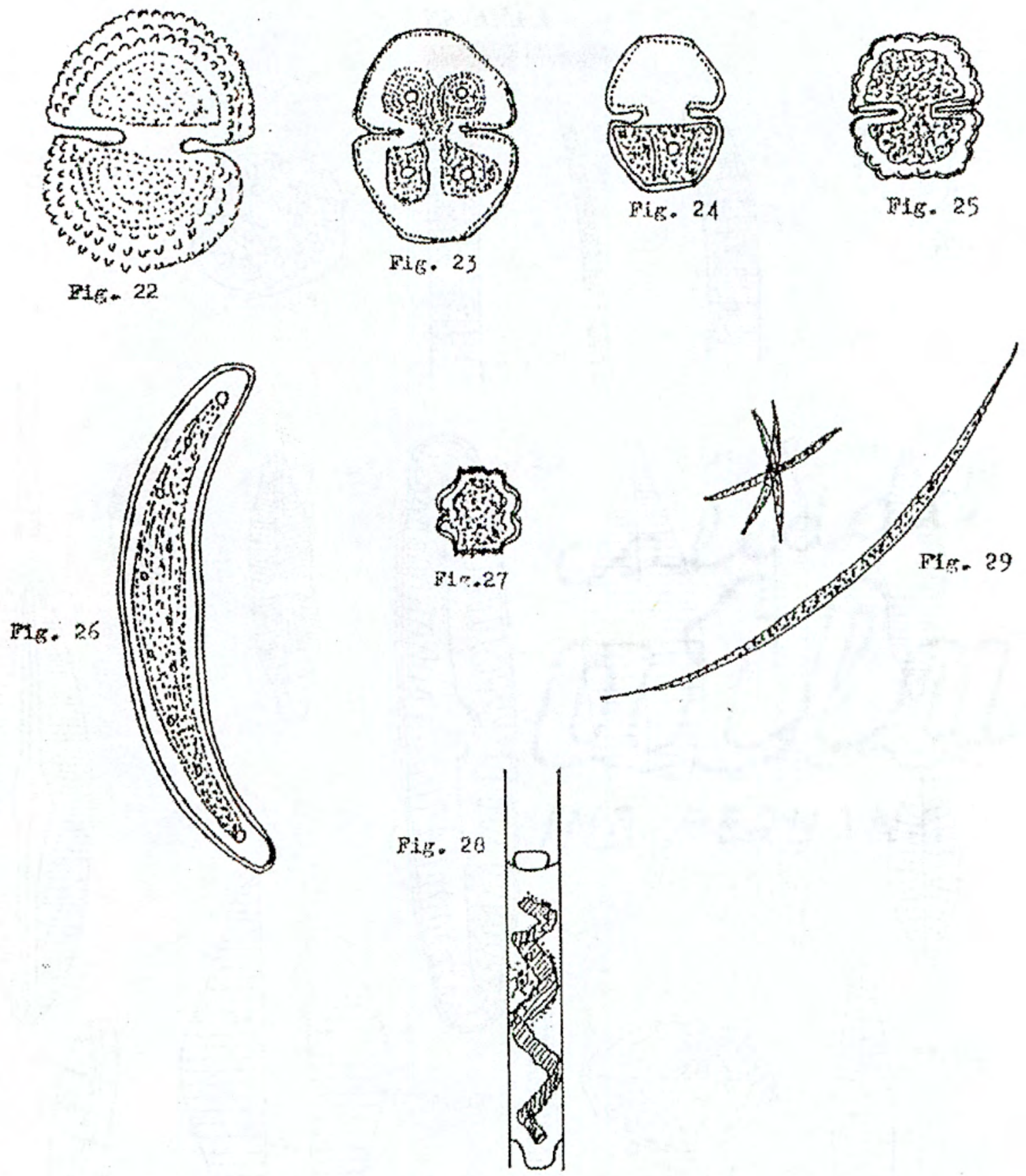


\section{UBICACIÓN GEOGRÁFICA DEL RÍO KAÑO}

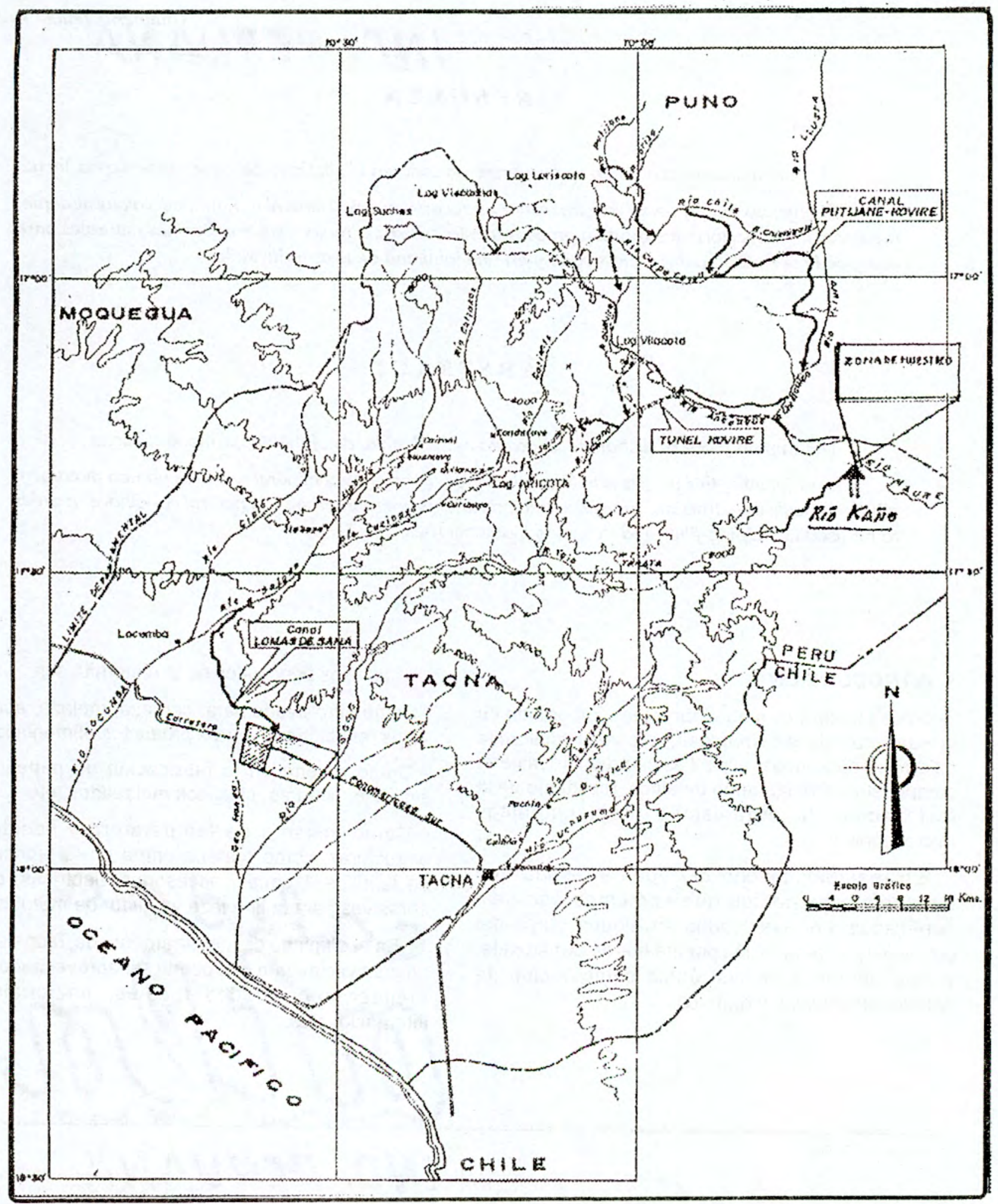

